\section{Comparison of two- and three-point sutures for advancing the levator aponeurosis in Asian eyelids}

YS Kim¹, JS Yoon ${ }^{2}$ and SY Jang ${ }^{1,3}$

\begin{abstract}
Purpose To compare the functional and cosmetic outcomes of two- and three-point sutures for advancing the levator aponeurosis in blepharoptosis surgery on Asians. Patients and methods This retrospective study examined 60 Asian patients with blepharoptosis who had undergone advancement of the levator aponeurosis: 34 patients (46 eyelids) had ptosis correction using the two-point suture technique and 26 patients (41 eyelids) had ptosis correction using the three-point suture technique. The postoperative marginal reflex distance (MRD1), lid height difference, and eyelid contour were evaluated.

Results Twenty-seven (79.4\%) of the 34 patients in the two-point group and 19 (73.1\%) of 26 patients in the three-point group had a postoperative MRD1 of $2-4 \mathrm{~mm}$, lids within $0.5 \mathrm{~mm}$ of each other, and a satisfactory eyelid contour; this difference was not significant. The rate of reoperation did not differ significantly between the two groups. Conclusion Two- and three-point sutures for advancing the levator aponeurosis were equally effective for correcting blepharoptosis in Asians.
\end{abstract}

Eye (2015) 29, 1181-1185; doi:10.1038/eye.2015.107; published online 26 June 2015

\section{Introduction}

A better understanding of the anatomy and physiology of the eyelid has led to refinements in blepharoptosis surgery. ${ }^{1}$ Patients with levator aponeurotic blepharoptosis most often require surgical repair, and anterior approaches such as a levator aponeurosis advancement ${ }^{2}$ and posterior repair involving resection of Muller's muscle $^{3-5}$ are effective in most cases.
Levator aponeurosis advancement for blepharoptosis is an effective procedure for establishing good eyelid position, with reported success rates from $70 \%$ to $>95 \% .^{6-11}$ In this procedure, the levator aponeurosis is usually sutured to the tarsus with three-point sutures. ${ }^{6,12-15}$ However, adjusting and fixing the levator aponeurosis on the tarsus at three different positions is relatively time and labor consuming. As the superior tarsus is narrow medially and laterally, much more dissection is needed to insert one additional suture. Recently, some clinicians have reduced the number of sutures used to fix the levator aponeurosis on the tarsus. $8,16-21$

However, most of the previous studies involved Caucasians; few reports have examined levator aponeurosis advancement in Asian patients. Therefore, this study compared the functional and cosmetic outcomes of two- and three-point sutures for advancing the levator aponeurosis in blepharoptosis surgery in Asians.

\section{Materials and methods}

A retrospective chart review was conducted of 60 patients with blepharoptosis seen at Soonchunhyang University Bucheon Hospital from March 2013 to December 2014 who underwent advancement of the levator aponeurosis by the same experienced oculoplastic specialist (SYJ): 34 patients (46 eyelids) with the two-point suture technique and 26 patients (41 eyelids) with the three-point suture technique. Exclusion criteria included previous eyelid surgery, concomitant surgery performed at the time of ptosis repair, and a follow-up period of $<3$ months.
${ }^{1}$ Department of Ophthalmology, Soonchunhyang University Bucheon Hospital, Soonchunhyang University College of Medicine,

Bucheon, Korea

${ }^{2}$ Department of Ophthalmology, Severance Hospital, Institute of Vision Research, Yonsei University College of Medicine,

Seoul, Korea

${ }^{3}$ Yonsei University Graduate School of Medicine, Seoul, Korea

Correspondence: SY Jang, Department of Ophthalmology, Soonchunhyang University Bucheon Hospital, Soonchunhyang University College of Medicine, 170 Jomaru-ro, Wonmi-gu, Bucheon 420-767, Korea Tel: +82 326216718 ; Fax: +82 326215018 . E-mail: ysyat01@naver.com

Received: 27 October 2014 Accepted in revised form: 30 April 2015 Published online: 26 June 2015 
This study adhered to the principles of the Declaration of Helsinki and was approved by the Institutional Review Board of the Soonchunhyang Bucheon Hospital, Soonchunhyang University College of Medicine.

\section{Outcome measures}

The marginal reflex distance (MRD1) is the distance between the inferior margins of the upper eyelid and the pupillary light reflex in the primary position of gaze. Levator function was assessed by measuring the total upper eyelid excursion from extreme down gaze to up gaze, while pressing over the patient's eyebrow to prevent action of the frontalis. The eyelid contour was judged from postoperative photographs. A successful surgical outcome was defined as a postoperative MRD1 of 2-4 mm, eyelid levels within $0.5 \mathrm{~mm}$ of one another, and satisfactory eyelid contour.

\section{Surgical technique}

The surgery was performed under local anesthesia. The skin crease was marked so that it was symmetric with that on the opposite side of the eyelid and 1-2 $\mathrm{ml}$ of $2 \%$ lidocaine with $1: 100000$ epinephrine was injected subcutaneously into the eyelid. The skin incision was made and deepened with scissors to the superior border of the tarsus through the orbicularis muscle, and extended medially and laterally. The dissection was directed upward posterior to the orbicularis muscle to expose the orbital septum, which was then opened widely to expose the preaponeurotic fat. The levator aponeurosis and Muller's muscle were dissected from the upper border of the tarsal plate and conjunctiva, until Whitnall's ligament was identified. The aponeurosis was measured for the desired amount of resection and fixed to the exposed tarsal border with two- or threepoint mattress sutures with 6-0 black Nylon (Ailee, Busan, Korea). In the three-point group, the inferior aponeurosis edge was fixed to the anterior tarsal surface using three horizontal mattress sutures: one of the sutures was placed at mid-pupil and medial and lateral sutures were placed at the midpoint between the first suture and each canthus. In the two-point group, two mattress sutures were placed vertically in two lines about $3 \mathrm{~mm}$ medially and laterally from the center of the pupil (Figure 1).

The sutures were adjusted according to eyelid position while sitting in the primary gaze position. The skin incision was sutured with 7-0 black Nylon (Ailee, Busan, Korea). Oral and topical antibiotics were administered routinely for 1 week postoperatively.

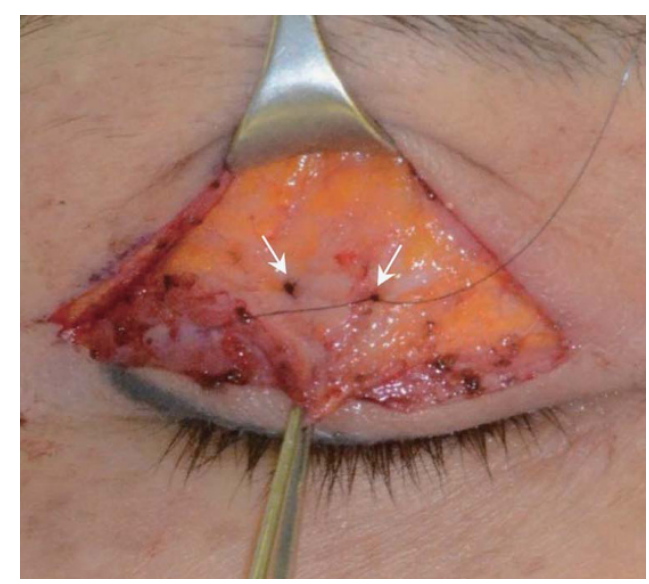

Figure 1 Intraoperative photograph of a two-point suture (white arrows) for advancing the levator aponeurosis.

\section{Data analysis and statistics}

The data were analyzed using SPSS (SPSS, Chicago, IL, USA). The Mann-Whitney $U$-test and $\chi^{2}$ test were used to compare values between the two groups. Unless otherwise indicated, the results are expressed as mean $\pm \mathrm{SD}$, and a value of $P<0.05$ was considered statistically significant.

\section{Results}

This study included 60 patients undergoing advancement of levator aponeurosis for blepharoptosis repair. The mean age of the patients in the two- and three-point groups was 61.2 (range 17-86) and 54.4 (range 9-81) years, respectively $(P=0.232)$. The respective mean postoperative follow-up was 4.3 (range 3-12) and 5.5 (range 3-17) months $(P=0.196)$. The proportion with involutional ptosis was higher in the two-point group $(88.2 \%)$ than the three-point group $(69.2 \%)$, but this difference was not significant $(P=0.068)$. Neither the mean preoperative MRD1 nor levator function differed significantly between the two groups $(P=0.993, P=0.136$, respectively; Table 1).

The criteria postoperative MRD1 of 2-4 mm, lids within $0.5 \mathrm{~mm}$ of each other, and satisfactory eyelid contour were met by $27(79.4 \%)$ of the 34 patients in the two-point group and $19(73.1 \%)$ of 26 patients in the three-point group; however, this difference was not significant (Table 2).

The postoperative MRD1 was $2-4 \mathrm{~mm}$ in 40 eyelids $(87.0 \%)$ in the two-point group. Of the six failures, two were $1.0 \mathrm{~mm}$ and four were $1.5 \mathrm{~mm}$. In the three-point group, the postoperative MRD1 was $2-4 \mathrm{~mm}$ in 31 eyelids $(75.6 \%)$. Of the ten failures, 2, 3, 4, and 1 were $0,0.5,1$, and $1.5 \mathrm{~mm}$, respectively. The two groups did not differ 
Table 1 Demographic characteristics of the 60 patients (87 eyelids) operated on for blepharoptosis

\begin{tabular}{lccc}
\hline Demographic characteristic & Two-point group & Three-point group & P-value \\
\hline Number of patients & 34 & 26 & - \\
Bilaterality & 12 & 15 & - \\
Number of eyes & 46 & 41 & $0.396^{\mathrm{a}}$ \\
Sex (male/female) & $12 / 22$ & $54.4 \pm 22.5(9-81)$ & $0.232^{\mathrm{b}}$ \\
Age (years) & $61.2 \pm 18.5(17-86)$ & & $0.068^{\mathrm{a}}$ \\
Cause & & 18 & \\
$\quad$ Involutional ptosis & 30 & 8 & $0.993^{\mathrm{b}}$ \\
Congenital ptosis & 4 & $-0.54 \pm 1.22(-3$ to 2$)$ & $0.136^{\mathrm{b}}$ \\
Preoperative MRD1 (mm) & $-0.52 \pm 1.06(-3$ to 2$)$ & $8.71 \pm 2.87(5-15)$ & \\
Preoperative LF (mm) & $9.18 \pm 2.35(5-16)$ & &
\end{tabular}

Abbreviations: LF, levator function; MRD1, marginal reflex distance. ${ }^{a}$ On the basis of $\chi^{2}$-test. ${ }^{b}$ On the basis of Mann-Whitney $U$-test.

Table 2 Outcomes of blepharoptosis surgery with two- and three-point sutures for advancing the levator aponeurosis

\begin{tabular}{lcrr}
\hline Outcome & Two-point group 34 patients (46 eyes) & Three-point group 26 patients (41 eyes) & P-value \\
\hline Successful surgical outcome & 27 patients $(79.4 \%)$ & 19 patients $(73.1 \%)$ & 31 eyes $(75.6 \%)$ \\
Postoperative MRD1; $2-4$ mm & 40 eyes $(87.0 \%)$ & 21 patients $(80.8 \%)$ & 0.565 \\
$\leq 0.5$ mm difference in eyelid height & 30 patients $(88.2 \%)$ & 38 eyes $(92.7 \%)$ & 0.173 \\
Satisfactory eyelid contour & 43 eyes $(93.5 \%)$ & 5 patients $(19.2 \%)$ & 0.605 \\
Clinical decision for reoperation & 4 patients $(11.8 \%)$ & 0.422 \\
\hline
\end{tabular}

Abbreviation: MRD1, marginal reflex distance. ${ }^{\mathrm{a}}$ On the basis of $\chi^{2}$-test.

significantly in having a postoperative MRD1 of $2-4 \mathrm{~mm}$ $(P=0.565)$.

The postoperative lid height difference was $\leq 0.5 \mathrm{~mm}$ in 30 patients $(88.2 \%)$ in the two-point group, with one of the four failures being in the bilateral group. The difference between the two sides was $1.0 \mathrm{~mm}$ in one bilateral and two unilateral cases, and $1.5 \mathrm{~mm}$ in one unilateral case. The lid height difference postoperatively was $\leq 0.5 \mathrm{~mm}$ in 21 patients $(80.8 \%)$ in the three-point group, with three of the five failures being in the bilateral group. The difference between the two sides was $1.0 \mathrm{~mm}$ in one unilateral and two bilateral cases, and $2.0 \mathrm{~mm}$ in one unilateral and one bilateral case. The two groups did not differ in having lids within $0.5 \mathrm{~mm}$ of each other $(P=0.422)$.

The eyelid contour, as judged from postoperative photographs, was satisfactory in 43 eyelids (93.5\%) in the two-point group. Of the three unsatisfactory eyelids, one was flat centrally, another was low medially with nasal webbing, and the other was triple fold. In the three-point group, the lid contour was satisfactory in 38 eyelids $(92.7 \%)$ with one low medially and two flat centrally. The two groups did not differ significantly in having a satisfactory eyelid contour $(P=0.605)$.

A decision to reoperate was made in four patients in the two-point group (14.3\%). Of the four, three had a $>0.5 \mathrm{~mm}$ difference in eyelid height and one had unsatisfactory eyelid contour. No recommendation for reoperation was made for three patients: one with a lid difference of $1.0 \mathrm{~mm}$ and two with a postoperative MRD1 of $1.5 \mathrm{~mm}$. The former patient showed $4 \mathrm{~mm}$ on the operated lid and $5 \mathrm{~mm}$ on the unoperated lid, and the latter two had a $0.5 \mathrm{~mm}$ difference and satisfactory eyelid contour. Three patients were satisfied with their result, and reoperation was not recommended. In the three-point group, a clinical decision for reoperation was made for five patients (19.2\%): three bilateral cases in whom each lid was similarly low, and two cases in whom the lid difference was $>0.5 \mathrm{~mm}$. No recommendation for reoperation was made in two patients with a lid difference of $1.0 \mathrm{~mm}$. The operated lids were higher than the unoperated lids in both patients who were satisfied with their result. The frequency of the clinical decision for reoperation was not significantly different in the two groups $(P=0.422)$.

\section{Discussion}

This study compared the surgical outcome of two- and three-point sutures for advancing the levator aponeurosis in Asian blepharoptosis patients. The results showed that the surgical outcomes of the two techniques were similar for correcting blepharoptosis in Asians using the following criteria: postoperative MRD1 of $2-4 \mathrm{~mm}$, lids 
within $0.5 \mathrm{~mm}$ of each other, and satisfactory eyelid contour. These criteria were met for $27(79.4 \%)$ of the 34 patients in the two-point group and 19 (73.1\%) of 26 patients in the three-point group; the difference was not significant. The frequency of reoperation did not differ significantly between the two groups. Different authors prefer a postoperative MRD1 of $2-4 \mathrm{~mm}^{18,22}$ or $2-4.5$ $\mathrm{mm}, 7,9,23$ and an acceptable difference in lid height above the center of the pupil of $0.5 \mathrm{~mm}^{16,18}$ or $1.0 \mathrm{~mm} \cdot{ }^{6,13,14,23-25}$ To compare the cosmetic outcome and patient satisfaction between the two groups, strict criteria for success were chosen and a satisfactory eyelid contour was included in the surgical outcome.

The surgical correction of blepharoptosis should be individualized based on the cause and amount of ptosis, the degree of levator function, and the patient's gender and race. Levator aponeurosis advancement for blepharoptosis has success rates of $70 \%$ to $>95 \%{ }^{6-11}$ As these studies all dealt with Caucasians, considering the differences in eyelid anatomy and surgical outcomes according to ethnicity, it is necessary to report the surgical outcomes of levator aponeurosis advancement in Asians. Because of the large orbicularis muscle, preaponeurotic fat, and narrower horizontal fissure, it might be more difficult to elevate the upper eyelid in Asians compared with Caucasians. This hypothesis is supported by Jang et $a{ }^{26}$ who investigated the relationship between the amount resected in a conjunctival Mullerectomy and lid elevation. They showed that the amount of lid elevation per $1 \mathrm{~mm}$ of conjunctival Mullerectomy was insufficient in Asians compared with Caucasians. Consequently, most oculoplastic surgeons in Korea use three- or four-point mattress sutures with 6-0 Nylon to fix the inferior aponeurosis edge to the anterior tarsal surface. However, adjusting and fixing the levator aponeurosis on the tarsus at three or four different positions is relatively time and labor consuming.

Indeed, much effort has been made to simplify this procedure. ${ }^{8,16-21} \mathrm{Liu}^{8}$ reported a technique that uses a single-suture aponeurotic tuck. Meltzer et al ${ }^{16}$ presented excellent results with an adjustable single suture. Lucarelli and Lemke ${ }^{17}$ published the first small-incision ptosis procedure and used a single suture predominantly, adding additional sutures as needed. Since that time, many surgeons have reported that this technique is simple and effective. ${ }^{18-21}$ In their case series, Jung and $\mathrm{La}^{19}$ obtained excellent surgical results for blepharoptosis repair through a small orbital septum incision with minimal dissection and a single fixation suture between the levator aponeurosis and tarsal plate in Asians. Unlike Jung and $\mathrm{La}^{19}$, we decided to reduce the number of sutures from three to two to simplify the traditional advancement of the levator aponeurosis. This led us to conduct this retrospective, comparative study.
However, we believe that a study comparing the onepoint and traditional techniques would be valuable.

We were concerned that decreasing the number of levator fixation sutures might increase the risk of recurrent ptosis and were concerned about eyelid contour problems. In recent studies, ${ }^{27,28}$ supratarsal fixation of the silicone rods at two points resulted in good patient outcomes in frontalis suspension surgery. Kim et al ${ }^{27}$ reported the functional centre of the upper eyelid, suggesting its clinical significance when performing ptosis surgery. They demonstrated that the outcome of silicone-rod surgery was excellent when the rod was fixed $4.4 \mathrm{~mm}$ nasal and $3.9 \mathrm{~mm}$ temporal from the functional eyelid centre. We believe that these results provide clinical evidence supporting our study. In this study, we placed the levator fixation sutures vertically in two lines about $3 \mathrm{~mm}$ medially and laterally from the center of the pupil in the two-point group (Figure 1). With this method, the postoperative eyelid contour was satisfactory in 43 eyelids (93.5\%) in the two-point group, and it was similar to the three-point group $(92.7 \%)$.

Permanence should be considered when judging the surgical outcome of ptosis repair. Concern that decreasing the levator fixation suture count might increase the risk of recurrence of ptosis is legitimate. Therefore, long-term follow-up is important. In this study, all patients were followed for at least 3 months. Frueh et al ${ }^{18} \mathrm{did}$ not report the follow-up time in their study comparing the efficacy of a new small-incision, single-suture ptosis procedure with that of a traditional ptosis surgery because all patients were discharged from care if lid position was adequate at 2 months. Doxanas ${ }^{29}$ followed 150 patients for 3-5 years and reported no case of late recurrence, which means that if patients were over or under corrected, this was evident 1 week after surgery. ${ }^{29}$ In our series, the time of reoperation in the two groups ranged from 1 day to 1 month after surgery.

In terms of time and simplicity, the two-point suture technique might be better than three-point suture technique. However, due to the study's retrospective design, there were no data on the operating time; therefore, we could not compare the time between the two groups. However, the two-point suture technique should be simpler and faster for adjusting and fixing the levator aponeurosis on the tarsus than the three-point suture technique, because the superior tarsus narrows medially and laterally, and much more dissection is needed to insert one additional suture.

In conclusion, this study showed that the functional and cosmetic outcomes of correcting blepharoptosis using the two- and three-point suture techniques were similar. Therefore, two- or three-point sutures for advancing the levator aponeurosis might be equally effective for correcting blepharoptosis in Asians. 


\section{Summary}

\section{What was known before}

- The levator aponeurosis is usually sutured to the tarsus with three-point sutures for correcting blepharoptosis in Asians.

\section{What this study adds}

- Two- or three-point sutures for advancing the levator aponeurosis might be equally effective for correcting blepharoptosis in Asians.

\section{Conflict of interest}

The authors declare no conflict of interest.

\section{Acknowledgements}

This study was supported by Soonchunhyang University Research fund.

\section{Disclosure}

The sponsor or funding organisation had no role in the design or conduct of this research.

\section{References}

1 Anderson RL, Beard C. The levator aponeurosis. Attachments and their clinical significance. Arch Ophthalmol 1977; 95(8): 1437-1441.

2 McCord CD Jr. An external minimal ptosis procedureexternal tarsoaponeurectomy. Trans Sect Ophthalmol Am Acad Ophthalmol Otolaryngol 1975; 79(5): 683-686.

3 Fasanella RM. Surgery for minimal ptosis: the FasanellaServat operation, 1973. Trans Ophthalmol Soc UK 1973; 93(0): 425-438.

4 Fasanella RM, Servat J. Levator resection for minimal ptosis: another simplified operation. Arch Ophthalmol 1961; 65: 493-496.

5 Putterman AM, Urist MJ. Muller muscle-conjunctiva resection. Technique for treatment of blepharoptosis. Arch Ophthalmol 1975; 93(8): 619-623.

6 Anderson RL, Dixon RS. Aponeurotic ptosis surgery. Arch Ophthalmol 1979; 97(6): 1123-1128.

7 Berlin AJ, Vestal KP. Levator aponeurosis surgery. A retrospective review. Ophthalmology 1989; 96(7): 1033-1036; discussion 1037.

8 Liu D. Ptosis repair by single suture aponeurotic tuck. Surgical technique and long-term results. Ophthalmology 1993; 100(2): 251-259.

9 McCulley TJ, Kersten RC, Kulwin DR, Feuer WJ. Outcome and influencing factors of external levator palpebrae superioris aponeurosis advancement for blepharoptosis. Ophthal Plast Reconstr Surg 2003; 19(5): 388-393.
10 Older JJ. Levator aponeurosis surgery for the correction of acquired ptosis. Analysis of 113 procedures. Ophthalmology 1983; 90(9): 1056-1059.

11 Shore JW, Bergin DJ, Garrett SN. Results of blepharoptosis surgery with early postoperative adjustment. Ophthalmology 1990; 97(11): 1502-1511.

12 Berke RN. Results of resection of the levator muscle through a skin incision in congenital ptosis. AMA Arch Ophthalmol 1959; 61(2): 177-201.

13 Jones LT, Quickert MH, Wobig JL. The cure of ptosis by aponeurotic repair. Arch Ophthalmol 1975; 93(8): 629-634.

14 Paris GL, Quickert MH. Disinsertion of the aponeurosis of the levator palpebrae superioris muscle after cataract extraction. Am J Ophthalmol 1976; 81(3): 337-340.

15 Wagar S, McMurray C, Madge SN. Transcutaneous blepharoptosis surgery-advancement of levator aponeurosis. Open Ophthalmol J 2010; 4: 76-80.

16 Meltzer MA, Elahi E, Taupeka P, Flores E. A simplified technique of ptosis repair using a single adjustable suture. Ophthalmology 2001; 108(10): 1889-1892.

17 Lucarelli MJ, Lemke BN. Small incision external levator repair: technique and early results. Am J Ophthalmol 1999; 127(6): 637-644.

18 Frueh BR, Musch DC, McDonald HM. Efficacy and efficiency of a small-incision, minimal dissection procedure versus a traditional approach for correcting aponeurotic ptosis. Ophthalmology 2004; 111(12): 2158-2163.

19 Jung Y, La TY. Blepharoptosis repair through the small orbital septum incision and minimal dissection technique in patients with coexisting dermatochalasis. Korean J Ophthalmol 2013; 27(1): 1-6.

20 Baroody M, Holds JB, Sakamoto DK, Vick VL, Hartstein ME. Small incision transcutaneous levator aponeurotic repair for blepharoptosis. Ann Plast Surg 2004; 52(6): 558-561.

21 Bernardini FP, de Conciliis C, Devoto MH. Mini-invasive ptosis surgery. Orbit 2006; 25(2): 111-115.

22 Goncu T, Cakmak S, Akal A, Karaismailoglu E. Improvement in levator function after anterior levator resection for the treatment of congenital ptosis. Ophthal Plast Reconstr Surg 2015; 31(3): 197-201.

23 Al-Abbadi Z, Sagili S, Malhotra R. Outcomes of posteriorapproach 'levatorpexy' in congenital ptosis repair. Br J Ophthalmol 2014; 98: 1686-1690.

24 Fox SA. Levator tucking. Ann Ophthalmol 1979; 11(3): 453-456.

25 Harris WA, Dortzbach RK. Levator tuck: a simplified blepharoptosis procedure. Ann Ophthalmol 1975; 7(6): 873-878.

26 Jang SY, Chin S, Jang JW. Ten years' experience with unilateral conjunctival mullerectomy in the Asian eyelid. Plast Reconstr Surg 2014; 133(4): 879-886.

27 Kim CY, Son BJ, Lee SY. Functional centre of the upper eyelid: the optimal point for eyelid lifting in ptosis surgery. Br J Ophthalmol 2015; 99(3): 346-349.

28 Buttanri IB, Serin D, Karslioglu S, Akbaba M, Ari S, Fazil K. Effect of suturing the silicone rod to the tarsal plate and the suture material used on success of frontalis suspension surgery. Ophthal Plast Reconstr Surg 2013; 29(2): 98-100.

29 Doxanas MT. Simplified aponeurotic ptosis surgery. Ophthalmic Surg 1992; 23(8): 512-515. 